$11-7-2006$

\title{
Ion transport and electrochemical tuning of Fermi level in single- wall carbon nanotube probed by in situ Raman scattering
}

\author{
S. Gupta \\ Missouri State University \\ J. Robertson
}

Follow this and additional works at: https://bearworks.missouristate.edu/articles-cnas

\section{Recommended Citation}

Gupta, S., and J. Robertson. "Ion transport and electrochemical tuning of Fermi level in single-wall carbon nanotube probed by in situ Raman scattering." Journal of applied physics 100, no. 8 (2006): 083711.

This article or document was made available through BearWorks, the institutional repository of Missouri State University. The work contained in it may be protected by copyright and require permission of the copyright holder for reuse or redistribution.

For more information, please contact BearWorks@library.missouristate.edu. 


\title{
Ion transport and electrochemical tuning of Fermi level in single-wall carbon nanotube probed by in situ Raman scattering
}

\author{
S. Gupta ${ }^{\text {a) }}$ \\ Department of Physics and Materials Science, Missouri State University, Springfield, Missouri 65897 \\ and Department of Electrical and Computer Engineering, University of Missouri, Columbia, \\ Missouri 65211

\section{J. Robertson} \\ Department of Engineering, University of Cambridge, Trumpington Street, Cambridge CB2 1PZ, \\ United Kingdom
}

(Received 28 September 2005; accepted 31 July 2006; published online 26 October 2006)

\begin{abstract}
In situ Raman spectroscopy technique was employed to investigate the ion transport process and to determine the concomitant electrochemical tuning of Fermi level in single-wall carbon nanotube. The variation of structural bonding in single-wall carbon nanotube bundle dipped in aqueous alkaline earth halide electrolyte such as $\mathrm{CaCl}_{2}$ with electrochemical biasing was monitored. It is because Raman can detect changes in $\mathrm{C}-\mathrm{C}$ bond length through radial breathing mode at $\sim 184 \mathrm{~cm}^{-1}$ that varies inversely with the nanotube diameter and the $G$ band at $\sim 1590 \mathrm{~cm}^{-1}$ that varies with the axial bond length. Consistent reversible and substantial variations in Raman intensity of both the modes induced by electrode potential point at the fine and continuous tuning (alternatively, emptying/depleting or filling) of the specific bonding and antibonding states. Qualitatively, the results were explained in terms of changes in the energy gaps between the one-dimensional van Hove singularities present in the electron density of states arising possibly due to the alterations in the overlap integral of $\pi$ bonds between the $p$ orbitals of the adjacent carbon atoms. We estimated the extent of variation of the absolute potential of the Fermi level and overlap integral $\left(\gamma_{0}\right)$ between the nearest-neighbor carbon atoms from modeling the electrochemical potential dependence of Raman intensity. Observations also suggest that the work function of the tube is larger for the metallic nanotubes in contrast to the simultaneously present semiconducting nanotubes. () 2006 American Institute of Physics. [DOI: 10.1063/1.2357839]
\end{abstract}

\section{INTRODUCTION}

Overwhelming interest in single-wall carbon nanotubes (SWNTs) - elongated members of fullerenes and cylindrical molecules of carbon-owes to their structural (molecular scale) and physical (optical, mechanical, chemical, and electronic) properties. Therefore, they serve as building blocks of nanoscience and nanotechnology. SWNTs are among the best candidates for a wide range of innovative ultrasmall functional units in ultrasensitive, low-energy consumption electronic and mechanical nanodevices ${ }^{1}$ such as field emission guns, ${ }^{2}$ catalyst supports, ${ }^{3}$ biosensors, ${ }^{4}$ tips for atomic force microscopes, ${ }^{5}$ and electromechanical devices such as actuators $^{6}$ and supercapacitors. ${ }^{7}$ Usage of SWNTs in these applications requires an understanding of the fundamental underlying physical mechanisms, which are inherently linked to their structural and, in turn, electronic structure properties. $^{8}$

Several characteristics of the electronic band structure of individual SWNTs, such as the energy separation between van Hove singularities and the density of states, have been obtained successfully employing recently developed experimental techniques in confocal micro-Raman spectroscopy, scanning tunneling microscopy, ${ }^{10}$ and fluorescence

\footnotetext{
${ }^{a)}$ Author to whom correspondence should be addressed; electronic mail: guptas@missouri.edu
}

methodology. ${ }^{11}$ In addition to these band profiles, information regarding absolute potential of energy levels of the states is known to be essential to nanodevice fabrication. The parameters of interest related to single-tube nanodevices include barrier height at a SWNT/metal junction, gate voltage to control doping of a semiconducting SWNT, threshold voltage for field emission SWNT electrodes, chemical reactivity of a single SWNT for biochemical sensors and energy storage substrates, etc. One of the electronic properties of SWNT known as work function reflects the absolute potential of the Fermi level of SWNT. Although several studies on the work function of carbon nanotubes have been conducted, these results do not coincide with one another. ${ }^{12}$ Furthermore, since control of valence electrons is crucial for nanoscale electronic devices, their doping behavior needs to be elucidated. Particular interest in the electronic properties of SWNTs seems to be complicated by their existence, either in metallic or semiconducting forms, depending upon their diameter, the wrapping vector, and torsion (collectively described as chirality). ${ }^{13}$

It has long been known that $\mathrm{C}-\mathrm{C}$ bonds in trans-polyacetylene ${ }^{14}$ (tr-PA) and graphite ${ }^{15}$ elongate/shorten when doped with electron donors/acceptors. The recent discovery of electrochemical actuation of SWNTs has brought renewed attention to these two systems. Since SWNTs possess outstanding electronic and mechanical properties, ${ }^{16,17}$ it 
is not surprising that their electromechanical properties are unusual. ${ }^{6}$ For example, one of the key applications of nanotubes based on electrochemical double layer (ECDL) (separates charges on an electrode from the ionic charges in solution) is an electromechanical actuator and it is in the growing list of existing actuating materials. ${ }^{18,19}$ A sheet of single-wall carbon nanotubes is dipped into an electrolyte and it expands or contracts if a voltage is applied between the sheet and a counterelectrode. A significant advantage of SWNTs for such studies lies in their high electrical conductivity, which obviates the need of metallic contacts for actuating devices, unlike in the case of polymers. ${ }^{20}$ Though it has been predicted that actuation is due primarily to changes in orbital occupation and band structure (quantum mechanical) with a secondary contribution arising from double-layer electrostatic effects (classical), ${ }^{24}$ the fundamental question of its operation at molecular level and its quantification is still rudimentary.

The reasons for the present study lie in the fact that doped SWNTs may represent a family of synthetic metals. $^{21,24}$ It is because doping reduces resistivity of SWNTs, but there is very little information available on the nature and quantification of the electronic band changes occurring due to ion transport. In this respect, the objectives of the present study are (a) to understand the actuation mechanism considering the solvation/hydration aspects, (b) to investigate the nature of electrochemical doping in carbon nanotubes, and (c) to determine quantitatively the electrochemical tuning of Fermi level and absolute potential. The advantage of spectroelectrochemical approach is that the electronic structure of SWNT can be closely probed while fine tuning its electrochemical potential or the Fermi level as suggested in Ref. 22. Furthermore, since the electrode potential (versus reference electrode) can in principle be converted into the absolute energy scale (versus vacuum level), we could estimate average values of the ionization potential and electronic affinity of single-walled nanotube bundles produced by Nanoledge, France. ${ }^{23}$

\section{EXPERIMENTAL DETAILS}

A self-supported SWNT bundled mat (hereinafter, SWNTm) was synthesized from single-wall nanotube soot (harvested from electrical arc discharge technique) obtained from Nanoledge, France, the details of which are described elsewhere. $^{23,24}$ They were typically obtained by vacuum filtration of nanotube suspension through a poly(tetrafluoroethylene) (PTFE) filter. The typical thickness of the SWNTm was $\sim 30-50 \mu \mathrm{m}$, a bulk density of $0.3-0.4 \mathrm{~g} / \mathrm{cm}^{3}$, and a four-probe conductivity of $5000 \mathrm{~S} / \mathrm{cm}$. This sheet was used as a working electrode (WE) in $5 \times 5 \mathrm{~mm}^{2}$ dimension with a mass of $0.75 \mathrm{mg}$. Typically, the SWNTm has either no preferred orientation or semiorientation. The largely bundled nanotube sheet was aligned along the surface and was examined using scanning electron microscopy (SEM, model JEOL 6340 FEGSEM) and high-resolution transmission electron microscopy (HRTEM, model JEOL JEM 4000EX, $400 \mathrm{kV}$ ). ${ }^{6}$

Raman spectra were measured in the backscattering configuration with a Renishaw double grating spectrometer (RM1000 series), using the $514.5 \mathrm{~nm}$ line of an $\mathrm{Ar}^{+}$laser
(Spectra-Physics model) as an excitation source with a few microwatts of incident power to avoid the thermal degradation with integration time of $60 \mathrm{~s}$. To integrate the electrochemical cell with a Raman spectrometer, a long working distance (LWD) objective lens of $50 \times$ was used (model Leica). Since the spectra were recorded using a LWD objective lens, the probed area was about $5 \mu \mathrm{m}^{2}$. The spectrometer slits were set at $100 \mu \mathrm{m}$, resulting in a resolution of $\sim 1.0 \mathrm{~cm}^{-1}$ or smaller in peak positions. The illuminating light was focused after each measurement in order to increase the throughput and also to keep the level of intensity the same throughout. All of the spectra were fitted using Jandel Scientific PEAKFIT software (v. 4.0) based on the Marquardt-Levenberg method. ${ }^{25}$

The electrochemical potential control with the Raman spectroscopy was analyzed using a Solartron 1287 electrochemical interface. ${ }^{7}$ Testing was carried out in the potentiostatic mode between -1.0 and $+1.0 \mathrm{~V}$ with steps of $0.1 \mathrm{~V}$ versus reference electrode [a standard $\mathrm{Ag} / \mathrm{AgCl}(4 M \mathrm{KCl})$ electrode and also known as saturated calomel electrode (SCE)], in a three-electrode single compartment electrochemical cell using argon deoxygenated aqueous electrolyte of alkaline earth halide, which is $0.1 M \mathrm{CaCl}_{2}$. The Raman spectra were measured at the end of each potential step, after waiting for the current to fall below $1 \mu \mathrm{A}$ to ensure quasiequilibrium conditions. The electrical contact was made using Pt wire that passed through electrical feedthroughs composed of alligatorlike stainless steel fittings. All of the chemicals were analytical grade supplied by Sigma-Aldrich and Alfa AEsar, and they were used as received. The electrolyte solutions were prepared using de-ionized (DI) water. The schematic of the experiment exhibiting the construction of artificial muscle (actuators having characteristics similar to human muscle, in that they are linear compliant elements which undergo reversible length changes in response to chemical stimuli) $^{26}$ from a SWNTm and the ongoing processes including redox reactions at the NT electrode/ electrolyte interface resulting in contraction/expansion is shown in Fig. 1.

\section{RESULTS AND DISCUSSION}

Resonant Raman spectroscopy has affirmed itself as a powerful method by studying electronic transitions in semiconductors. It has been applied to the investigation of the quantum properties of electrons and phonons in carbon nanotubes and to identify the nanotube diameter $\left(d_{t}\right)$ in a sample containing a mixture of chiralities. ${ }^{27}$ The selectivity to the Raman response for the nanotube band structure is due to the one-dimensional (1D) quantum confinement as $k$ vectors can take continuous values only in the axial direction. Therefore, by selecting excitation wavelength of $514.5 \mathrm{~nm}\left(E_{L}\right.$ $=2.41 \mathrm{eV}$ ), we preferentially probe semiconducting tubes based on the zone-folding scheme, matching $v_{s}^{3} \leftrightarrow c_{s}^{3}$ transition, while strictly metallic nanotubes $\left(v_{m}{ }^{1} \leftrightarrow c_{m}{ }^{1}\right)$ are analyzed when a higher excitation wavelength of $632.8 \mathrm{~nm}$ $\left(E_{L}=1.96 \mathrm{eV}\right)$ is used, recognized as a resonant effect. ${ }^{28,29}$ Nevertheless, it is worth noting that pristine single-wall nanotube sheet consists of both semiconducting and metallic 
(a)
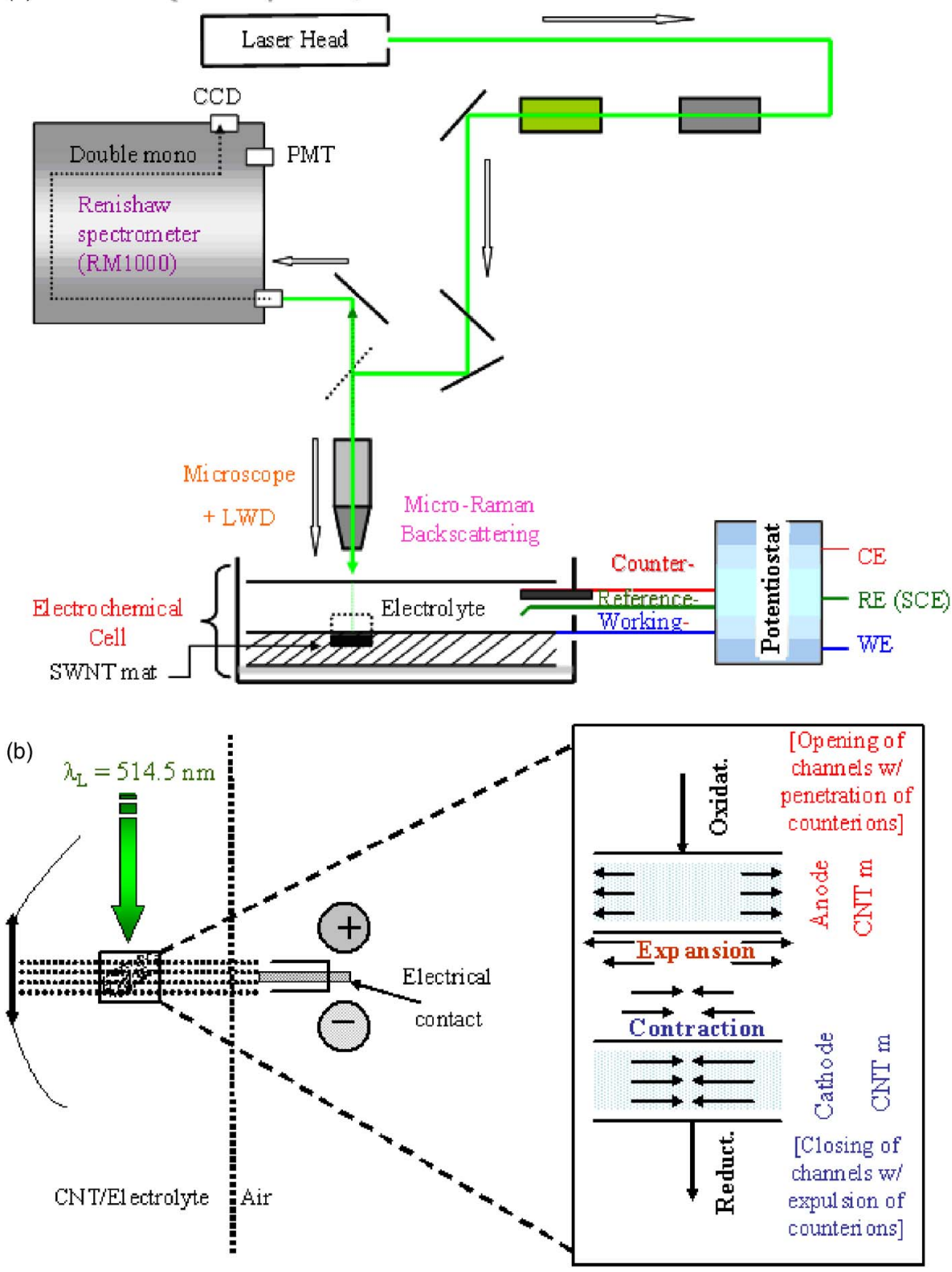

FIG. 1. (Color online) (a) Schematic of the experimental setup of in situ Raman spectroelectrochemistry: Counter, reference, and working refer to three electrodes in a single compartment electrochemical cell. (b) Schematic illustration of the experiment exhibiting the construction of artificial muscle from a sheet of SWNT and the ongoing process at the CNT/ electrolyte interface shown in dotted square in (a). nanotubes and the Raman spectrum from either wavelength therefore represents a convolution of both depending upon the diameter distribution. Moreover, assignment of the vibrational features in the Raman spectrum of SWNT is carried out according to Ref. 29. The pronounced signatures of interest in a typical Raman spectrum of SWNT shown in Fig. 2 are low frequency radial breathing mode (RBM), disorderinduced mode ( $D$ band), doubly degenerate tangential displacement mode (TDM or $G$ band), and second-order $D$ mode ( $G^{\prime}$ band). The $G$ band results from the splitting of intralayer stretching mode in graphite possessing $E_{2 g 2}$ symmetry and is due to curvature-induced rehybridization usually located at $\sim 1560 \mathrm{~cm}^{-1}\left(T_{2}\right.$ mode $)$ and $\sim 1593 \mathrm{~cm}^{-1}\left(T_{3}\right.$ mode). They seem to be quite narrow $\left[\left(\Gamma_{\mathrm{RBM}}, 5-7 \mathrm{~cm}^{-1}\right.\right.$ and $\left.\left.\Gamma_{G}, 4-5 \mathrm{~cm}^{-1}\right)\right]$ and sharp, indicating the high uniformity of the SWNTs and the low level of impurities. Through an empirical relation, the diameter of the nanotubes $\left(d_{t}\right)$ is scaled with frequency position of RBM, $\nu_{\mathrm{RBM}}=234 / d_{t}(\mathrm{~nm}) .{ }^{29}$ The constant value of 234 was calculated using local density ap-

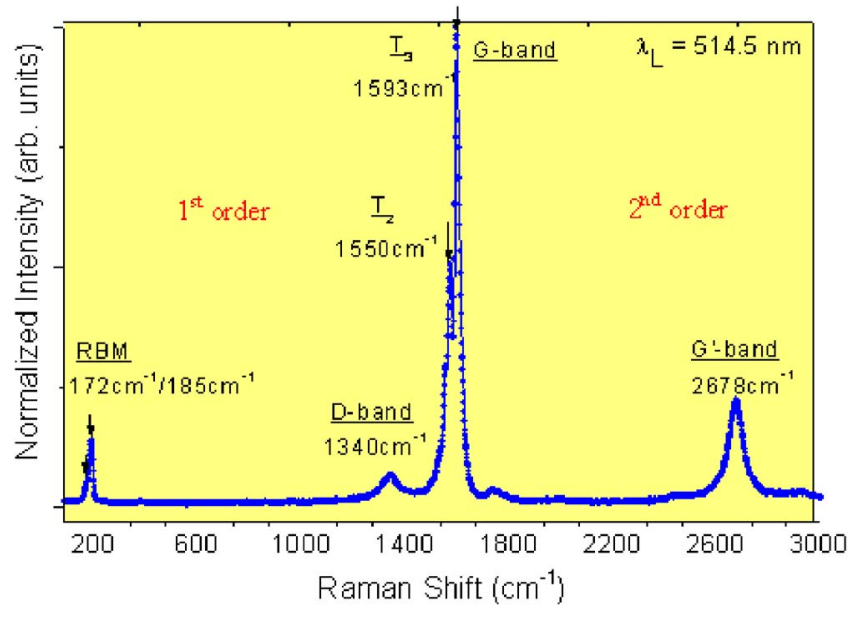

FIG. 2. (Color online) Typical Raman spectrum of single-wall nanotube either isolated adsorbed on Au electrode in solution or of a mat showing characteristic bands both in first order and second order. 
proximation and it was chosen because it takes into account the intertube interaction. ${ }^{29}$ The calculated values of $d_{t}$ ranged between 1.14 and $1.37 \mathrm{~nm}$. $D$ band is a unique feature of $s p^{2}$-bonded carbon materials, arising due to in-plane substitutional heteroatoms, vacancies, grain boundaries, or other defects and size effects, all of which lower the translational symmetry and violate the momentum conservation principle. ${ }^{6}$ SWNTm is described as compositionally disordered material since it contains a mixture of semiconducting and metallic nanotubes. Consistent with the previous discussion, for this range of nanotube diameters at excitation energy of $2.41 \mathrm{eV}$, we are ascertained that metallic tubes with $0.9<d_{t}<1.1$ and semiconducting nanotubes with diameters $1.2<d_{t}<1.6$ are in resonance due to the allowed interband transitions: $E_{11}^{M}$ and $E_{33}^{S}$.

The mechanistic details of the electrochemical charging process via double-layer formation on the high surface area walls of SWNTs leading to nanoscale actuation and modification in electronic structure were probed using in situ Raman spectroscopy under open circuit voltage (OCV) and electrochemical bias control. This is because Raman can detect changes in $\mathrm{C}-\mathrm{C}$ bond length $\left(d_{\mathrm{C}-\mathrm{C}}\right)$, because the RBM at $\sim 190 \mathrm{~cm}^{-1}$ varies inversely with the nanotube diameter, and the $G$ band at $\sim 1590 \mathrm{~cm}^{-1}$ varies with the axial bond length. In addition, the intensities of both the modes vary with the occupation of bonding and antibonding states due to charge transfer (quantum chemical). However, before we commenced the charge transfer experiments, we made an attempt to test if there was any change of the prominent Raman bands (both in position and intensities) when the SWNT sheet is immersed in aqueous electrolytes. Absolutely no change in frequency position and only a minimal change in intensity were observed. Corresponding loss in the Raman scattering intensities of these modes in liquid perhaps originates from light scattering by the solution. Figure 3 displays a series of first-order Raman spectral signatures (both the RBM and $G$ band) of SWNT in $0.1 M \mathrm{CaCl}_{2}$ electrolyte, as a function of electrochemical bias ranging from -1.0 to $+1.0 \mathrm{~V}$ with an interval of $0.1 \mathrm{~V}$ with respect to SCE. Notice that the Raman spectra shown in Fig. 3 are the normalized spectra with respect to the $T_{3}$ band in the corresponding liquid, and for all of the electrolytes all of the spectra were measured under the same conditions. High voltages $(|V|$ $\gg 1 \mathrm{~V}$ ) were avoided because they can (a) produce tiny bubbles at the surface of the working electrode in the solution, which can hardly be seen by the naked eye and may hamper light scattering and (b) lead to delamination and cracking [similar to atomic cleavage in highly oriented pyrolytic graphite (HOPG)] accompanied by gas $\left(\mathrm{Cl}_{2}\right.$, for instance, and/or $\mathrm{O}_{2}$ ) bubbles, the formation of which is represented as

$$
\begin{aligned}
& 2 \mathrm{Cl}^{-} \rightarrow \mathrm{Cl}_{2}(g)+2 e^{-}, \quad E_{0}=1.1 \mathrm{~V}(\mathrm{vsSCE}), \\
& 2 \mathrm{H}_{2} \mathrm{O} \rightarrow \mathrm{O}_{2}(g)+4 \mathrm{H}^{+}+4 e^{-}, \quad E_{0}=0.99 \mathrm{~V}(\mathrm{vsSCE}) .
\end{aligned}
$$

Figure 4 shows the temporal behavior of the Raman intensity observed at frequency of RBM, $\nu_{\mathrm{RBM}}=185 \mathrm{~cm}^{-1}$ on a Pt electrode in an electrolyte solution. The potential was stepped or scaled between 0 and $+0.6 \mathrm{~V}$ (vs $\mathrm{Ag} / \mathrm{AgCl}$ ). It
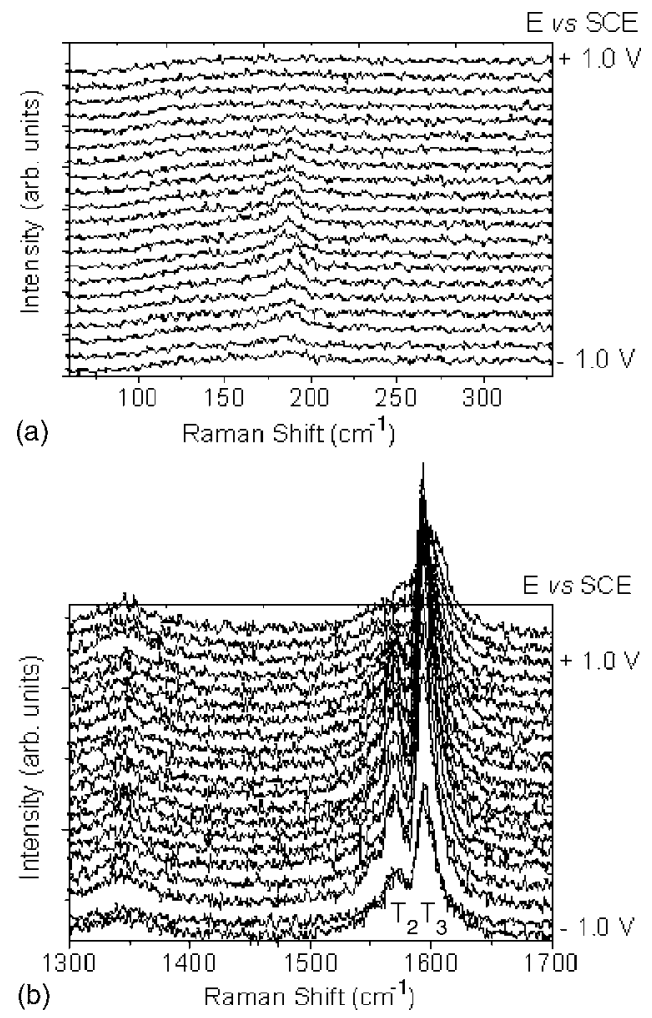

FIG. 3. Shown is one of the representative examples of in situ Raman spectra with $\lambda_{L}=514.5 \mathrm{~nm}$ of SWNT on polycrystalline Pt electrode in aqueous $0.1 M \mathrm{CaCl}_{2}$ electrolyte: (a) radial breathing mode (RBM) and (b) tangential displacement mode $(G)$ under electrochemical control relative to reference electrode [saturated calomel electrode (SCE)] from -1.0 to $+1.0 \mathrm{~V}$ in $0.1 \mathrm{~V}$ increments from bottom upwards.

shows that the intensity was dependent upon the electrochemical potential and was changed within $1 \mathrm{~s}$, and it recovered to the initial values at the same potential step. This result proves that the change in the Raman intensity caused by the electrode potential modulation is fast enough and shows superior reversibility. The Fermi level of SWNT may be aligned to that of a Pt electrode within the time span. The reversibility also implies that no significant chemical reaction and deformation occurred on SWNT during this electrochemical doping process by controlling the potential. This particular observation also tallies with cyclic voltammogram. ${ }^{6}$

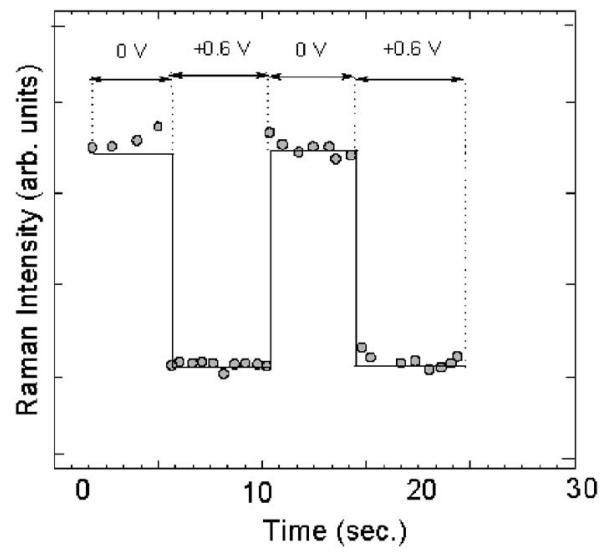

FIG. 4. Time course of the Raman intensity for the $\nu_{\mathrm{RBM}}\left(=185 \mathrm{~cm}^{-1}\right)$ band. Potential was stepped between 0 and +0.6 V. $T_{3}\left(=1593 \mathrm{~cm}^{-1}\right)$ mode also shows similar behavior. 

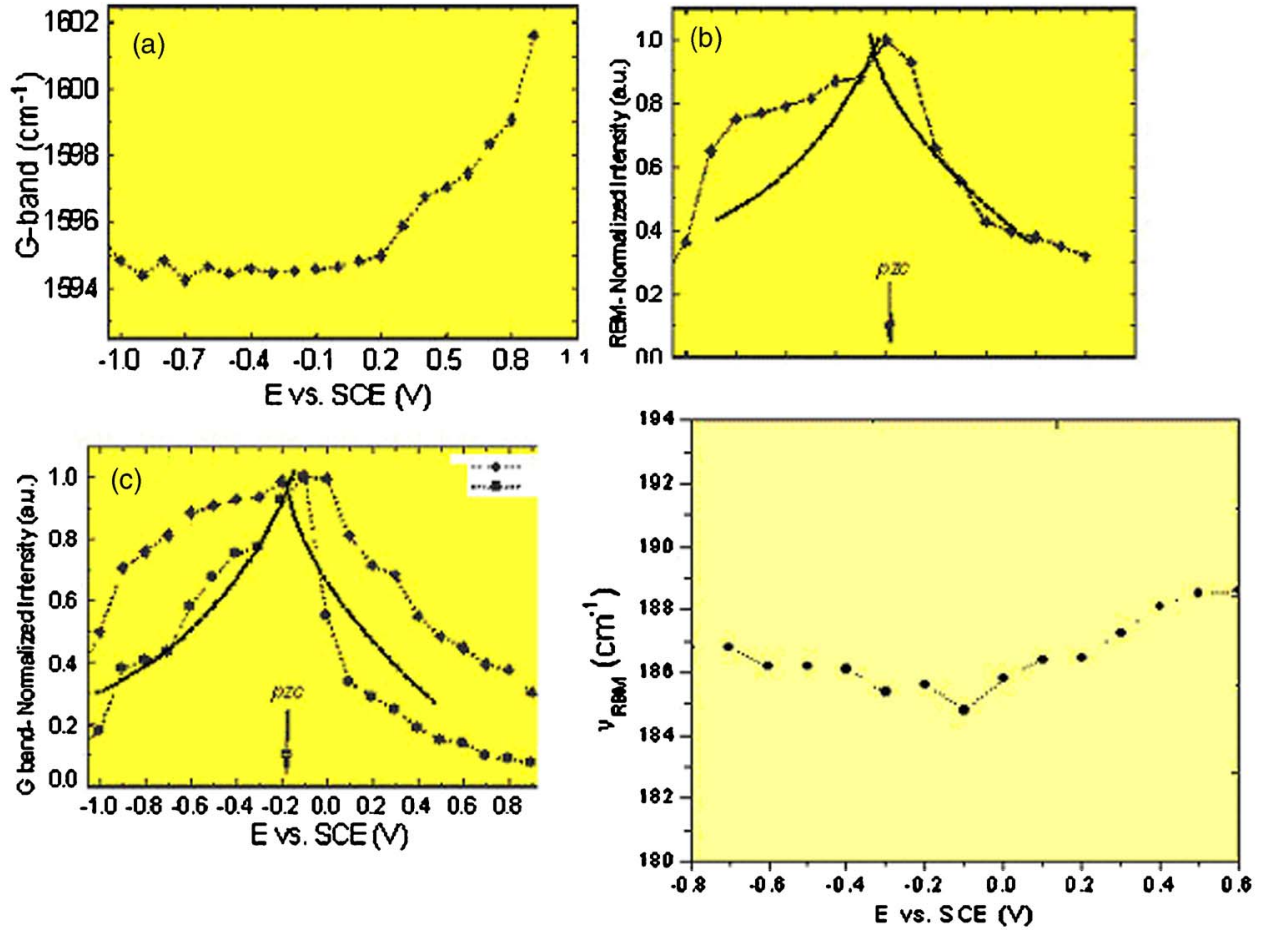

FIG. 5. (Color online) Shown is the variation of (a) Raman frequency position of the tangential displacement mode: $G$ band, Raman intensity of the (b) radial breathing mode and (c) tangential displacement mode (TDM): $G$ band (both $T_{2}$ and $T_{3}$ modes), and (d) Raman frequency position of the radial breathing mode (RBM).
Physically speaking, if the SWNTm is polarized electrochemically (either anodic or cathodic), some of the primary effects to be considered are as follows: (1) this process either softens or hardens $\mathrm{d}_{\mathrm{C}-\mathrm{C}}$ and, in turn, affecting the frequencies of the vibrational modes of interest (e.g., RBM and $G$ band) and it subsequently alters the resonance condition; (2) it changes the filling or emptying of electronic states of these tubes and once again modifies the resonance condition and; (3) a possibility of electrochemical-induced perturbation such as solvation/hydration and hydrostatic pressure on nanotubes which may have an effect on energy levels affecting the resonance condition is certainly not overlooked. ${ }^{30}$

Qualitative inspection of the spectroelectrochemical Raman data in Fig. 3 reveals some of the noteworthy features: (a) a drop in the intensities of RBM, $T_{2}$, and $T_{3}$; (b) effect on the position of the bands is more pronounced for the positive potentials than that of negative; (c) Raman spectra at the highest potential look similar to the amorphous or disordered carbon, likely due to resonance effect; (d) broadening or even disappearance of the RBM band accompanies the effect on splitting of TDM and; (e) since electrochemical charging is a reversible process, i.e., the changes occurring at cathodic potential excursion are similar to albeit not identical, occurring at anodic potential excursion. Following these effects in mind, we analyzed our Raman spectroelectrochemical data quantitatively in terms of intensity and frequency of the pronounced features with respect to bias to garner information about electronic structure.

Figure 5 shows potential dependence of various Raman spectral features such as position of $G$ band and RBM [(a) and (d)] and their intensity [(b) and (c)]. The intensity of metallic and semiconducting tubes observed in the experiment in Fig. 5(c) is particularly shown through $T_{3}$ band. Relatively broad potential dependence was observed because of the simultaneous presence of both the metallic and semi- conducting tubes. The gradual potential dependence is one of the characteristics of a bundled SWNT. Another characteristic of the electrochemical response is the potential at which the Raman intensity is maximum, $\phi_{0}$, becomes increasingly positive as $\nu_{\mathrm{RBM}}$ increases. The $\phi_{0}$ of metallic tubes is relatively positive as $\nu_{\mathrm{RBM}}$ increases as well with respect to semiconducting tubes.

As far as the variation in the frequency position of the bands is concerned, the change in RBM is found to be $\sim 3 \mathrm{~cm}^{-1}$ which is larger than the experimental uncertainties of $\pm 0.5 \mathrm{~cm}^{-1}$ [Fig. 5(d)], implying that the counterions usually tend to stay on the surface of the nanotube bundles, rather than inserting themselves in the nanochannels or the open network and even interstitial spaces of nanotube bundles described schematically in Ref. 18, unlike the effects observed in graphite intercalation compounds (GICs). ${ }^{18}$ However, the observed slight increase in the position of the $T_{3}$ band [Fig. 5(a)] by a few wave numbers, i.e., by about $2-10 \mathrm{~cm}^{-1}$, with increase in applied potential is attributed to reversible anodic oxidation of the tube walls. An upshift in the $T_{3}$ band is a consequence of stiffening on axial bond length under compressive stress [Fig. 5(a)]. ${ }^{6}$ It is apparent from our findings that the electron injection (electrochemical reduction) resulted in much smaller changes in the $T_{3}$ position than for the hole injection (electrochemical oxidation). Also, the intensity/frequency shift is not proportional to the applied potential, but there is an abrupt change at ca. $+0.5 / 0.6 \mathrm{~V}$ for both the modes. Nonetheless, shift in vibration frequencies expected as a result of change in physical dimension of SWNT expected on electrochemical charging is still debatable. ${ }^{18}$

Lorentzian deconvolution analyses shown in Figs. 5(b) and 5(c) xhibit a monotonic reversible variation for both the $\mathrm{RBM}$ and the $\mathrm{G}$ band peaking at slightly negative potentials of $\sim-0.1 /-0.2 \mathrm{~V}$ (vs SCE), depending upon the band. These 
results tally quite closely with those reported by An et al., ${ }^{31}$ Kavan et al., ${ }^{32}$ and Duesburg ${ }^{33}$ for the metallic nanotubes probed using $1.96 \mathrm{eV}$ excitation radiation. In spite of the decrease (reversible) in the intensities of both the modes, this variation shown in Figs. 5(b) and 5(c) depicts that the electron and hole injection across the $\pi-\pi^{*}$ bands into the SWNTm is uniform but not ideally bipolar or amphoteric in nature. This implies that hole injection seems to be more facile than that of electron injection. The overall decrease in intensity of these modes matches the results on chemical doping, but the electrochemical charging offers fine tuning of the electronic structure of SW nanotubes.

Another aspect of intensity variation of these bands is its shape, where the trend has a maximum [Figs. 5(b) and 5(c)], similar to that observed for strain/stress dependence on potential, in electrocapillary measurements on electrodes of activated carbon and exfoliated graphite materials. ${ }^{18}$ This response is expected if surface changes due to double-layer charging provide the mechanical stresses that elongate the material. Alternatively, if electrostatic effects dominate, the electrode dimension would be a minimum at the potential of zero charge (pzc), and either positive or negative charge injection would result in approximately equivalent contraction or expansion. Hereby, the observed pzc for $0.1 M \mathrm{CaCl}_{2}$ electrolyte solution is slightly negative and corresponds to the pzc for the basal plane of graphite. ${ }^{18}$ This observation is contrary to the one proposed in Ref. 18, where pzc is much higher $(+0.27 /+0.56 \mathrm{~V})$. The implication is that quantum chemical effects play a lesser role than the Coulombic effects for the carbon nanotube sheets, in contrast to the theoretical prediction and experimental observations based on charge transfer complexes in graphite and conducting polymers. ${ }^{18}$

The electronic spectra of single-walled nanotube are usually dominated by van Hove singularities (vHSs), manifestation of the 1D structure. The location of Fermi energy $E_{F}$ with respect to these singularities can be tuned by chemical (alkali metals, acids, and halogens) or electrochemical doping. Doping response in bulk materials is complicated by the presence of metallic and semiconducting tubes and by diameter and chirality dispersion, both of which imply a distribution of initial work functions. Further complications arise from tube-tube interactions in bundles or ropes. The results are discussed in terms of rigid band model ${ }^{34}$ where doping shifts $E_{F}$ without affecting the band structure. We assumed that in undoped samples, all of the tubes have the same work function, and that $E_{F}$ is initially near the middle of the gap or pseudogap of semiconducting and metallic tubes, respectively. We also assumed that doping is spatially uniform, with no energy barriers between metallic and semiconducting tubes. We find that this simplest model gives consistent results for the Fermi level shift $\left(\Delta E_{F}\right)$ upon doping. Using the experimentally determined $\Delta E_{F}$ values as input, the evolution of Raman spectra with doping can be simply explained by the variation in resonance conditions, with no evidence for diameter-selective doping as recently proposed. The distinctive utilization of Raman scattering technique is that each tube type in bulk samples has a unique Raman resonance behavior, due to diameter and symmetrydependent interband transitions. Doped SWNTs lose reso-

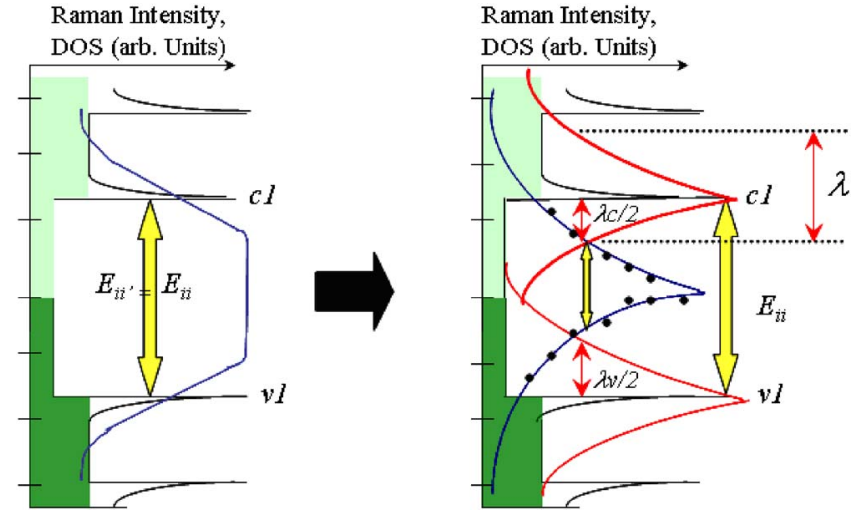

FIG. 6. (Color online) Shown is a general schematic of (a) energy level diagrams of single-walled carbon nanotubes under zero bias and (b) corresponding Raman scattering intensity variation considering the broadening in respective conduction $\left(\lambda_{c}\right)$ and valence $\left(\lambda_{v}\right)$ bands under electrochemical potential control. In (b) $\lambda$ is halved because it is represented as a half-width at half maximum.

nance via quenching of interband absorption by the shift of $E_{F}$, which is again different for individual tube type. Thus the interpretation of Raman spectra from bulk-doped samples is not straightforward, usually reflected in the Raman intensity.

The schematic of variation in resonance condition caused by electrochemical doping/undoping on the SWNTm electrode in aqueous electrolyte is shown in Fig. 6. The electrochemical anodic and cathodic polarizations of SWNTm caused depletion of the valence band and filling of the conduction band, respectively. The changes in the electronic density, which lead to bleaching of the electronic transition between vHSs in the visible region and the near infrared region (NIR), produce a decrease in resonant Raman intensity. In addition, the potentials where bleaching began were considered to reflect the energy levels of vHS.

Based on the aforementioned model of electrochemical doping or ion transport in SWNTm, the full width at half maximum (FWHM) of the potential dependence of the Raman intensity should reflect an energy separation between van Hove singularities, $E_{i i}$ in the present system (see Fig. 7). However, $E_{i i}^{\prime}$ are much smaller than those of corresponding $E_{i i}$. Figure 7 (right) shows potential dependence of the RBM intensity of metallic tube $\left(\nu_{\mathrm{RBM}}=172 / 185 \mathrm{~cm}^{-1}\right)$, superimposed on their electronic structure. The potential dependence is rather broad and is due to the mixed semiconducting and metallic nanotubes with FWHM of $0.6-1.1 \mathrm{~V}$, which is larger than those of isolated nanotubes. In addition, solvation may also alter the energy distribution of the electronic states of SWNT at the solution/electrode interface. The simultaneous presence of both types of nanotubes besides intertube interactions leads to somewhat broadened electronic bands. Additionally, the position of singularity peak depends upon the tube diameter and chirality, which has considerable distribution reflected in experimentally observed inevitably broad bands.

In general, electronic states of the molecules in solution acquire a specific distribution due to the vibration-rotation interaction among molecules and the surrounding solvent molecules. The Gaussian distribution associated with widths 


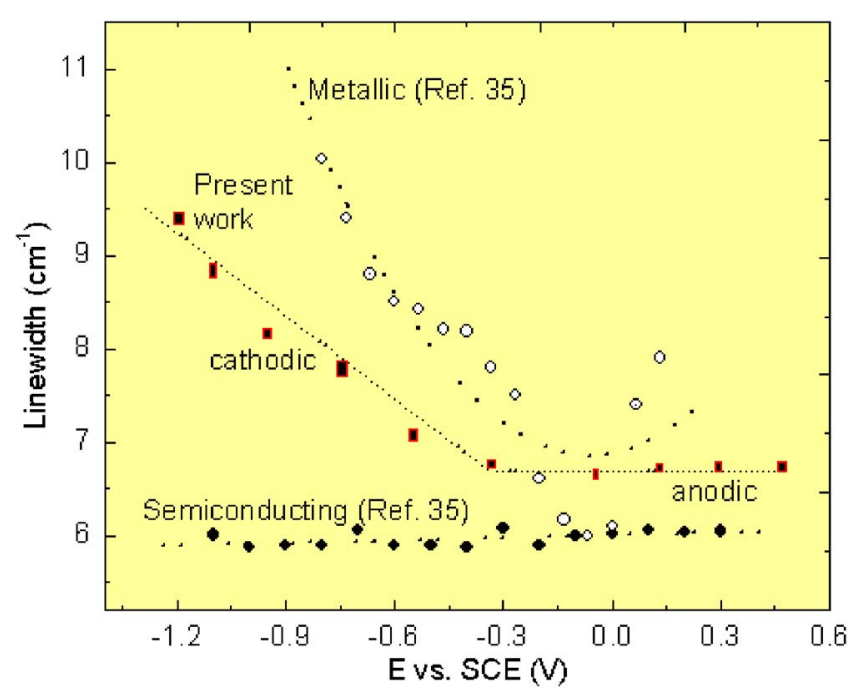

FIG. 7. (Color online) Shown is the variation of relation between observed $\nu_{\text {RBM }}$ position and absolute potential, $\phi_{0}$. The dashed fits are taken from Ref. 35 .

of approximately $0.6-1.1 \mathrm{eV}$ often provides a rough estimate of the density of the electronic states of molecules at the electrochemical interface. If one assumes that the energy levels of SWNT on a Pt electrode possess such distributions due to solvation, this broadened conduction (valence) band may allow the process of filling (depletion) of the electronic states at more positive (negative) electrochemical polarization, resulting in behavior shown in Fig. 8. In the present case, $E_{i i}^{\prime}$ depends upon both the $E_{i i}$ and the widths of the energy distribution of the states in conduction and valence bands; $\lambda_{c} / 2$ and $\lambda_{v} / 2$, respectively. Considering significant contribution of vHS in conduction and valence bands to the optical bleaching process, $E_{i i}^{\prime}$ is expected to display a smaller value than $E_{i i}$ as follows: $E_{i i}^{\prime}=E_{i i}-\left(\lambda_{c} / 2\right.$ and $\left.\lambda_{v} / 2\right) / 2$. Observed differences between $E_{i i}^{\prime}$ and $E_{i i}$ in the present system were in the range of $0.8-1.1 \mathrm{eV}$. These results indicate that the electrochemical potential dependence of Raman scattering intensity can be used to determine the position of the

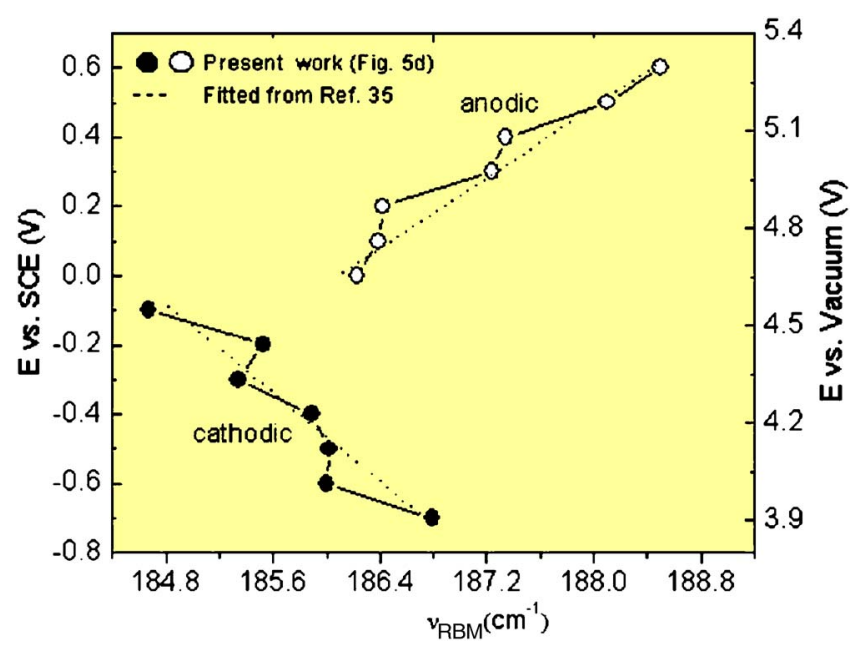

FIG. 8. (Color online) Variation of full width at half maximum for semiconducting and metallic nanotubes under electrochemical potential control for the $\mathrm{CaCl}_{2}$ electrolyte. For SWNT bundle the linewidth values lie intermediate. electronic states of a SWNT bundle at the interface. We proposed that the potential, at which the Raman intensity is at maximum, $\phi_{0}$, reflects the energy level of the center position between vHs, i.e., the Fermi level of bundled SWNTs.

Recalling Fig. 8 for discussion, it presents a plot of the variation of $\nu_{\mathrm{RBM}}$ for cathodic and anodic parts of the nanotubes and $\phi_{0}$. The dashed fits in Fig. 8 are from Refs. 12 and 35 . The basic tenet was to make an analogy with the isolated metallic and semiconducting nanotube studies reported in Ref. 34 and to deduce similar information. Diameterdependent shift of the work function is a feature of SWNTs and we detected a linear relation as shown in Fig. 6. The present findings suggest that chemical reactivity and electron emitting properties of SWNTs should have significant structural dependence. Currently there is no concrete explanation for this phenomenon and the qualitative comparisons between the charge transfer due to ion transport and the shift of the Fermi level are not currently available. The contribution of the $\sigma-\pi$ mixing in SWNT may be a possible factor for the alteration of the work function.

Here, the average values of work function at negative and positive scans reflect the energy level of the center position between vHSs, i.e., the Fermi level of an isolated SWNT. The work function of SWNT can be correlated to the absolute potential proposed by Kazaoui et al. ${ }^{36}$ It is known that electrochemical potential versus SCE is related to absolute potential relative to the vacuum level. Therefore, electrochemical method served to be the most reliable technique, unlike photoemission and others. Potential scale, $V_{\text {abs }}$ (versus vacuum level) $=V$ (vs NHE), which is equal to $4.44 V$, derived by Trasatti, ${ }^{37}$ and therefore, $V(\mathrm{vs} \mathrm{Ag} / \mathrm{AgCl})=V$ (vs NHE) $-0.197 \mathrm{~V}$, exhibited in Fig. 8. The values for SWNT observed in the present investigation are estimated from the dotted line in Fig. 8. The lines determined from the average of the values of anodic and cathodic scans afforded a simple relation between $\nu_{\mathrm{RBM}}$ and the work function $\Phi$, which is given by $\Phi=|e| V_{\mathrm{abs}}$, and it follows a simple relation $\Phi$ $=2.0 \times 10^{-2} \nu_{\mathrm{RBM}}+1.13$.

Figure 9 shows potential dependence of FWHM of RBM mode, $\Gamma_{\mathrm{RBM}}$. It is clear that the $\Gamma_{\mathrm{RBM}}$ is the specific behavior for metallic tubes adopted from Ref. 35, which can be analogized with the present behavior observed at cathodic (negative) potentials in Fig. 9. Such spectral broadening was observed rather weakly at anodic (positive) potentials for semiconducting nanotubes. These results apparently suggest that the electron doping contributes to the changes in the $\Gamma_{\mathrm{RBM}}$. The cause of the broadening of the RBM is partly ascribed to the adsorption of cation and salvation/hydration to negatively charged SWNTs surface, altering the distribution of the electronic states. Concerning the behavior of water molecules when placed in direct contact with the ion (both cation and anion), the difference between how water binds to cation and anion becomes important. The water molecules surrounding a cation tend to avoid each other, whereas it binds to anion. The free hydrogen is favorably oriented to associate with other molecules and form network in the hydration shell. ${ }^{38}$ Interaction between negatively charged SWNT, ions, and solvent molecules in surrounding media may be one of the possible origins for linewidth broadening 
of RBM. In addition to these extrinsic effects, some of intrinsic effects on the structural change may also be anticipated.

Although above mentioned factors are important for linewidth broadening, additional contribution such as free electrons in metallic tubes is equally important. For instance, it was shown that the chemical doping from halogens ( $p$ type) and alkali metals ( $n$ type) to SWNT bundles results in the increase of the optical absorption background attributed to Drude-type absorption. ${ }^{39}$

Here, we discuss the overlap integral determination by mathematical modeling of the Raman intensity which is. ${ }^{40}$

$$
I(\omega)=\sum_{\omega_{\mathrm{ph}}}\left(A \sum_{i=1}^{15} \sum_{d} \frac{1}{d^{2}} \frac{p(d)}{\left[\left(E_{i i}-E_{L}\right)^{2}+\Gamma_{e}^{2}\right]\left\{\left[E_{i i}-\left(E_{L}-\hbar \omega_{\mathrm{ph}}\right)\right]^{2}+\Gamma_{e}^{2}\right\}}\right) \frac{\Gamma}{4\left(\omega-\omega_{\mathrm{ph}}\right)^{2}+\Gamma^{2}},
$$

where $p(d) \approx e^{\left(d-d_{0}\right)^{2} / 2 \sigma^{2}}$ corresponds to diameter distribution with a cross section of $0.08 \mathrm{~nm}, 1 / d^{2}$ is the contribution from the joint density of states (JDOS) (electronic), $\Gamma_{e}$ is a broadening parameter $=0.05 \mathrm{eV}, E_{i i}=2 i \gamma_{0} a_{\mathrm{CC}} / d+\delta E$ (not too far from the Fermi level) and $i=1,2,4,5, \ldots$ (semiconducting); $i=3,6, \ldots$ (metallic); $\delta E=0.2 \mathrm{eV}$ (due to bundling), $\gamma_{0}=2.73$, i.e., $p p \pi$ overlap integral with $a_{\mathrm{CC}}=1.42 \AA, E_{L}$ $=2.41 \mathrm{eV}, \omega_{\mathrm{ph}}$ is the phonon frequency (for RBM it is $=234 / d_{t}$ ), and $\Gamma$ (full width at half maximum) is the phonon damping constant. Parameter $A$ contains all three matrix elements related to electron radiation and electron-phonon interaction Hamiltonians which are assumed to be constant. ${ }^{40}$ A typical diameter distribution will result in quasicontinuum of electronic transitions for both semiconducting and metallic SWNTs. The diameter enters in the above mentioned equation rather explicitly and through the diameter dependence of the transition energies $E_{i i}$. We find somewhat different values for the 1.26 and $1.36 \mathrm{~nm}$ diameter nanotubes and they were 2.7 and $2.8 \mathrm{eV}$. The apparent shift between the maxima and the transition energy $E_{i i}$ of the mean diameter is a direct consequence of the $1 / d_{t}$ enhancement of the Raman signal by smaller-diameter tubes. For semiconducting resonance maxima, $E_{i i}$ determined from the best-fit values for $\gamma_{0}$ are $E_{22}=1.3 \mathrm{eV}$ and $E_{44}=2.6 \mathrm{eV} \quad\left(d_{t}=1.3 \mathrm{~nm}\right)$ and $E_{22}$ $=1.2 \mathrm{eV}$ and $E_{44}=2.4 \mathrm{eV}\left(d_{t}=1.45 \mathrm{~nm}\right)$. The broadening parameter determined from our fit is $\hbar \gamma=33 \mathrm{meV}$ and is somewhat larger than previously reported. ${ }^{41}$ Constant $A$ is obtained by normalizing the $T_{3}$ peak intensity for zero bias condition and it was kept constant throughout the fitting. Although the summation was done for electronic transitions from $i=1-15$, the primary contribution to the Raman intensity comes from $i=4$ with a partial contribution from $i=3$. The difference in $\gamma_{0}$ value is found to be around $0.12 \mathrm{eV}$ (equivalently, $2.73 \mathrm{eV}$ ) less than that of zero bias condition from the best fit of the data to Eq. (2). We argue that the electrochemical bias (cathodic, in particular) alters only the value of $\gamma_{0}$, which in turn changes $E_{i i}$, analogous to changing resonance condition.

Usually, the Raman intensity from SWNT electrode is a sum of two contributions, one from the surface layer with $\gamma_{0}=\gamma(v)$ and the other from the underlying unaffected bulk with $\gamma_{0}=2.73 \mathrm{eV}$. To avoid additional parameters, these two contributions were taken to be equal while fitting the Raman intensities. Experimental data for the finite cathodic bias were fitted using Eq. (2) with only one adjustable parameter, $\gamma(v)$. The dotted lines in curves 5(b) and 5(c) show a calculated line shape with $\gamma(v=-0.10 \mathrm{~V}--0.20 \mathrm{~V})$ providing $2.61-2.60 \mathrm{eV}$. The agreement is reasonable and a slight discrepancy is due to the broadened energy bands of mixed semiconducting and metallic nanotubes as pointed out several times.

Figure 9 shows the variation of overlap integral $\gamma(v)$ with applied bias. A small change in $E_{i i}$ affects the RBM and $T_{3}$ band intensity substantially. Qualitatively, the variation of $\gamma_{0}$ under cathodic bias suggests the following explanation. With negative bias applied to the SWNT, the cations $\mathrm{Ca}^{2+}$ form a space charge double layer around the nanotubes which affect the overlap integral of the $\pi$ orbitals on adjacent carbon atoms separated by $a_{\mathrm{C}-\mathrm{C}}=0.142 \mathrm{~nm}$. This effect would undoubtedly depend on the size of cations, which is the case in our present study. We found that the effect using $\mathrm{Na}^{+}(0.19 \mathrm{~nm})$ is larger than that of $\mathrm{Ca}^{2+}(0.25 \mathrm{~nm})$. We believe that large anionic size is responsible for not observing the effect under anodic bias $\left(\mathrm{Cl}^{-}=0.36 \mathrm{~nm}\right)$. It is also tempting to correlate the decrease in $\gamma$ to the observed ex-

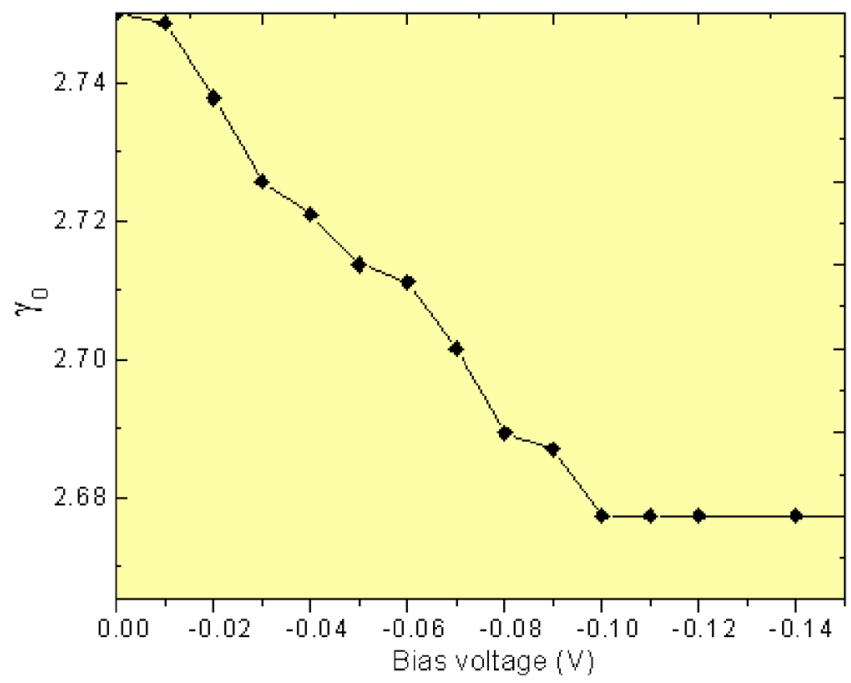

FIG. 9. (Color online) Variation of overlap integral $\left(\gamma_{0}\right)$ vs electrochemical potential with respect to reference electrode [saturated calomel electrode (SCE)] for $0.1 M \mathrm{CaCl}_{2}$ electrolyte. 
pansion of the nanotubes along the length under the applied bias. More theoretical work is needed to complement and to quantify this effect on the relative ionic sizes and to correlate the changes in electronic structure and actuator action.

\section{CONCLUSIONS}

In summary, we have conducted the charge transfer dynamics at the surface of the nanotubes using in situ Raman spectroelectrochemistry for $\mathrm{CaCl}_{2}$ aqueous electrolyte. The Raman spectra as a function of electrochemical bias were analyzed in terms of intensity, frequency, and Fermi level shift. The possibility of tuning the Fermi energy level of SWNTs by ions of different redox potentials allowed selective filling or emptying/depleting of density of states, in addition to electrostatic effect. The Fermi level shift was estimated using mathematical modeling of Raman intensity and it was $\sim 0.12 \mathrm{eV}$ as an upper bound, affecting the resonance condition. The interpretation of these experiments is complicated by the fact that mats consist of both semiconducting and metallic nanotubes and the charge transfer included hopping between the nanotubes and/or bundles. Therefore, the evolution of Raman spectra upon electrochemical doping is well accounted for by changes in Raman resonance conditions. We found no evidence that the doping is selective with respect to the tube diameter or metallic and semiconducting character. The present results in terms of absolute potential provided important information for future applications of single-walled carbon nanotubes in nanoelectronic devices. Furthermore, these findings are encouraging as they propose a step forward and may ultimately offer a strategy for materials selection and electrochemical device performance optimization.

\section{ACKNOWLEDGMENTS}

The authors wish to thank S. Hoebanx and Dr. J. Fraysse from Nanoledge, France for supplying the single-wall carbon nanotube mat used in the present study and Dr. M. Hughes (from University of Cambridge, UK) for useful discussions.

\footnotetext{
${ }^{1}$ M. Terrones, F. Banhart, N. Grobert, J.-C. Charlier, H. Terrones, and P. M. Ajayan, Phys. Rev. Lett. 89, 075505 (2002); F. Banhart, Nano Lett. 1, 329 (2001).

${ }^{2}$ M. M. J. Treacy, T. W. Ebbesen, and J. M. Gibson, Nature (London) 381, 678 (1996).

${ }^{3}$ B. Gao, A. Kelinhammes, X. P. Tang, C. Bower, Y. Wu, and O. Zhou, Chem. Phys. Lett. 307, 153 (1999); J. Liu et al., Science 280, 1253 (1998).

${ }^{4}$ J. Li et al., Nano Lett. 3, 597 (2003).

${ }^{5}$ C. L. Cheung, J. H. Hafner, and C. M. Lieber, Proc. Natl. Acad. Sci. U.S.A. 97, 3809 (2000); Y. Lin, W. Yantasee, and J. Wang, Front. Biosci. 10, 492 (2005)

${ }^{6}$ S. Gupta, M. H. Hughes, A. H. Windle, and J. Robertson, J. Appl. Phys 95, 2038 (2004); S. Gupta, Diamond Relat. Mater. 15, 378 (2006)

${ }^{7}$ M. Hughes, M. S. P. Shaffer, A. C. Renouf, C. Singh, G. Z. Chen, D. J. Fray, and A. H. Windle, Adv. Mater. (Weinheim, Ger.) 14, 382 (2002).

${ }^{8}$ J.-C. Charlier and Ph. Lambin, Phys. Rev. B 57, R15037 (1998).

${ }^{9}$ G. S. Duesberg, I. Loa, M. Burghard, K. Syassen, and S. Roth, Phys. Rev.
}

Lett. 85, $5436(2000)$

${ }^{10}$ J. W. G. Wildöer, L. C. Venema, A. G. Rinzler, R. E. Smalley, and C. Dekker, Nature (London) 391, 59 (1998); T. W. Odom, J. L. Huang, P. Kim, and C. M. Lieber, ibid. 391, 62 (1998).

${ }^{11}$ M. J. O'Connell et al., Science 297, 593 (2002).

${ }^{12}$ D. Lovall, M. Buss, E. Graugnard, R. P. Andres, and R. Reifenberger, Phys. Rev. B 61, 5683 (2000); S. Suzuki, C. Bower, Y. Watanabe, and O. Zhou, Appl. Phys. Lett. 76, 4007 (2000); M. Shiraishi and M. Alta, Carbon 39, 1913 (2001); J. P. Sun, Z. X. Zhang, S. M. Hou, G. M. Zhang, Z. N. Gu, X. Y. Zhao, W. M. Liu, and Z. Q. Xue, Appl. Phys. A: Mater. Sci. Process. 75, 479 (2002).

${ }^{13}$ C. L. Kane and E. J. Mele, Phys. Rev. Lett. 78, 1932 (1997).

${ }^{14}$ Y. Wada, in New Horizon in Low-Dimensional Electron Systems, Physics and Chemistry of Material with Low-Dimensional Structures, edited by $\mathrm{H}$. Aoki, M. Tsukada, M. Schluter, and F. Levy, (Kluwer Academic, Boston, 1991), pp. 415-432.

${ }^{15}$ M. S. Dresselhaus and G. Dresselhaus, Adv. Phys. 51, 2 (2002).

${ }^{16}$ M. M. J. Treacy, T. W. Ebbesen, and J. M. Gibson, Nature (London) 381, 678 (1996).

${ }^{17}$ M. R. Falvo, G. J. Curry, R. M. Taylor, V. Chi, F. P. Brooks, S. Washburn, and R. Superfine, Nature (London) 389, 582 (1997).

${ }^{18}$ R. H. Baughman et al., Science 284, 1340 (1999).

${ }^{19}$ J. E. Hubner, N. A. Fleck, and M. F. Ashby, Proc. R. Soc. London, Ser. A 453, 2185 (1997).

${ }^{20}$ T. W. Ebbesen, H. J. Lezec, H. Hiura, J. W. Bennett, H. F. Ghaemi, and T. Thio, Nature (London) 382, 54 (1996).

${ }^{21}$ M. S. Dresselhaus and G. Dresselhaus, in Light Scattering in Graphite Intercalation Compounds, Topics in Applied Physics Series Vol. 53, edited by M. Cardona and G. Güntherodt (Springer-Verlag, Berlin, 1982), p. 3.

${ }^{22}$ S. Kazaoui, N. Minami, H. Kataura, and Y. Achiba, AIP Conf. Proc. 544, $400(2000)$

${ }^{23}$ See http//:www.nanoledge.com

${ }^{24}$ S. Gupta, M. Hughes, A. H. Windle, and J. Robertson, Diamond Relat. Mater. 13, 1314 (2003).

${ }^{25}$ D. W. Marquardt, J. Soc. Ind. Appl. Math. 11, 431 (1963).

${ }^{26}$ S. Ashley, Sci. Am. July issue, 53 (2003).

${ }^{27}$ A. Jorio, R. Saito, J. H. Hafner, C. M. Lieber, M. Hunter, T. McClure, G. Dresselhaus, and M. S. Dresselhaus, Phys. Rev. Lett. 86, 1118 (2001)

${ }^{28}$ A. M. Rao et al., Science 275, 187 (1997).

${ }^{29}$ M. S. Dresselhaus and P. C. Eklund, Adv. Phys. 49, 705 (2000)

${ }^{30}$ S. Reich, C. Thomsen, and P. Ordejón, Phys. Rev. B 65, 153407 (2002); J. Sandler, M. S. P. Shaffer, A. H. Windle, M. P. Halsall, M. A. MontesMorán, C. A. Cooper, and R. J. Young, ibid. 67, 035417 (2003).

${ }^{31}$ C. P. An, Z. V. Zardeny, Z. Iqbal, G. Spinks, R. H. Baughman, and A. Zakhidov, Synth. Met. 116, 411 (2001).

${ }^{32}$ L. Kavan, P. Rapta, L. Dunsch, M. J. Bronikowski, P. Willis, and R. E. Smalley, J. Phys. Chem. B 105, 10764 (2001).

${ }^{33}$ G. S. Duesburg (unpublished).

${ }^{34}$ W. Zhou, J. Vavro, N. M. Nemes, J. E. Fischer, F. Borondics, K. Kamarás, and D. B. Tanner, Phys. Rev. B 71, 205423 (2005).

${ }^{35}$ K. Murakoshi and K.-I. Okazaki, Electrochim. Acta 50, 3069 (2005); K.-I. Okazaki, Y. Nakato, and K. Murakoshi, Phys. Rev. B 68, 035434 (2003).

${ }^{36}$ S. Kazaoui, N. Minami, N. Matsuda, H. Kataura, and Y. Achiba, Appl. Phys. Lett. 78, 3433 (2001)

${ }^{37}$ S. Trasatti, J. Electroanal. Chem. Interfacial Electrochem. 52, 313 (1974); R. Parsons, in Standard Potentials in Aqueous Solution, edited by A. J. Bard, R. Parsons, and J. Jordan (Dekker, New York, 1985).

${ }^{38}$ W. H. Robertson and M. A. Johnson, Annu. Rev. Phys. Chem. 54, 173 (2003).

${ }^{39}$ B. Ruzicka, L. Degiorgi, R. Gaal, L. Thien-Nga, R. Basca, J. P. Salvetat, and L. Forro, Phys. Rev. B 61, R2468 (2000).

${ }^{40}$ S. Ghosh, A. K. Sood, and C. N. R. Rao, J. Appl. Phys. 92, 1165 (2002); K.-I. Okazaki, Y. Nakato, and K. Murakoshi, Phys. Rev. B 68, 035434 (2003); J.-C. Charlier and Ph. Lambin, ibid. 57, R15037 (1998).

${ }^{41}$ A. M. Rao, P. C. Eklund, S. Bandow, A. Thess, and R. E. Smalley, Nature (London) 388, 257 (1997); G. Sun, M. Nicklaus, and M. Kertesz, Encyclopedia of Nanoscience and Nanotechnology (Dekker, New York, 2004), p. 3605. 